\title{
Electronic Structure of Arsabenzene: Microwave Spectrum, Dipole Moment, and Nuclear Quadrupole Coupling Constants
}

\author{
Robert P. Lattimer, Robert L. Kuczkowski, ${ }^{1}$ Arthur J. Ashe ili, \\ AND A. L. MeINZER \\ Deparlment of Chemistry, University of Michigan, Ann Arbor, Michigan 48104
}

\begin{abstract}
The microwave spectrum of arsabenzene was analyzed; $a$ dipole transitions were observed. The following rotational constants were obtained; $A=4871.03 \pm 0.18 \mathrm{MHz}, B=2295.87$ $\pm 0.01 \mathrm{MHz}, C=1560.10 \pm 0.01 \mathrm{MHz}$. The dipole moment was $1.10 \pm 0.04 \mathrm{D}$. The nuclear quadrupole coupling constants due to the ${ }^{75} \mathrm{As}$ nucleus were $\chi_{a a}=-186.4 \pm 0.1 \mathrm{MHz}$, $\chi_{b b}=43.5 \pm 0.2 \mathrm{MHz}, x_{c c}=142.9 \pm 0.2 \mathrm{MHz}$, and the asymmetry parameter, $\eta=0.533$ \pm 0.002 . Analysis of the quadrupole coupling constants indicated that the population of the $4 p$ orbitals on arsenic decrease in the order $n_{a}>n_{b}>n_{c}$.
\end{abstract}

\section{INTRODUCTION}

Although pyridine has been a familiar compound to chemists for many years, the heavier Group $\mathrm{V}$ congeners phosphabenzene, arsabenzene, stibabenzene, and bismabenzene have only recently been synthesized (1-3). Investigations of the physical, structural, and electronic properties of this series of compounds should provide information useful for interpreting and testing theories of bonding and aromaticity. Previous studies on arsabenzene include a structure determination by electron diffraction (4) which indicated a planar structure, a short $\mathrm{As}-\mathrm{C}$ bond length and $\mathrm{C}-\mathrm{C}$ bond lengths typical for aromatic compounds. A preliminary report of the photoelectron spectrum of arsabenzene along with theoretical calculations has provided a tentative assignment of its highest filled molecular orbitals (5). CNDO ( $6 a)$ and ab initio (6b) MO investigations of its electronic structure have also been reported. Our investigation contributes data obtained from the analysis of the microwave spectrum of arsabenzene including the rotational constants, the molecular dipole moment, and the ${ }^{75} \mathrm{As}$ nuclear quadrupole coupling constants.

\section{EXPERIMENTAL DETAILS}

Microwave transitions were measured at room temperature with an $80 \mathrm{kHz}$ Starkmodulated spectrometer using both oscilloscope and recorder display. Uncertainties in the measured frequencies were estimated at $\pm 0.10 \mathrm{MHz}$. The preparation of the sample has been previously reported (1).

${ }^{1}$ Author to whom correspondence can be sent.

${ }^{2}$ Alfred P. Sloan Fellow, 1972-76. 
TABLE I. Ground-state Transition Frequencies of Arsabenzene (in MHz).

\begin{tabular}{|c|c|c|}
\hline Transition & $\underline{v}_{0}^{v_{0}(o b s)^{a}}$ & $\Delta v^{b}$ \\
\hline${ }^{3} 03^{-4} 04$ & 14272.77 & 0.02 \\
\hline${ }^{3} 22^{-4} 23$ & 15316.88 & -0.01 \\
\hline${ }^{3} 21^{-4} 22$ & 16466.47 & 0.05 \\
\hline${ }^{4} 23^{-5} 24$ & 18977.66 & -0.01 \\
\hline${ }^{4} 32^{-5} 33$ & 19604.75 & 0.07 \\
\hline${ }^{4} 31^{-5} 32$ & 19918.52 & -0.04 \\
\hline${ }^{4} 13^{-5} 14$ & 20350.58 & -0.01 \\
\hline${ }^{4} 22^{-5} 23$ & 20895.47 & -0.03 \\
\hline${ }^{5} 15^{-6} 16$ & 20193.16 & -0.02 \\
\hline${ }^{5} 05^{-6} 06$ & 20397.37 & 0.01 \\
\hline${ }^{5} 24^{-6} 25$ & 22536.33 & -0.03 \\
\hline $5_{33^{-6}} 34$ & 23525.19 & -0.02 \\
\hline${ }^{5} 42^{-6} 43$ & 23583.00 & 0.13 \\
\hline $5_{41}-642$ & 23637.03 & -0.23 \\
\hline${ }^{5} 23^{-6} 24$ & 25220.24 & -0.15 \\
\hline
\end{tabular}

\footnotetext{
$a_{\text {Hypothetical unsplit frequencies which would occur in the absence }}$ of quadrupole interaction.$$
b_{v_{0}}(o b s)-v_{0}(c a l c) \text {. }
$$

Ground state spectrum. The structural parameters from the ED study (4) were used to predict the microwave spectrum. That study correctly predicted a $\mu_{a}$ dipole spectrum with rotational constants within 1-3 $\mathrm{MHz}$ of our final values. Hence in spite of a dense, somewhat weak spectrum, the assignment of the ground vibrational state was relatively easy. The transitions were identified initially by their quadrupole hyperfine splittings and the expected direction of their Stark shifts. Fifteen $R$-branch $a$-type transitions were assigned; they are listed in Table I. The derived rotational constants and moments of inertia are given in Table II. The rotational constants were determined by a leastsquares fit of the hypothetical unsplit frequencies $\left(\nu_{0}\right)$ which would occur in the absence of quadrupole interaction. The uncertainties listed are twice the standard deviations. No excited vibrational states or isotopically substituted species were assigned. Figure 1 depicts arsabenzene in its principal axes system.

Quadrupole hyperfine structure. All transitions were found to be split into several components due to the presence of the ${ }^{75} \mathrm{As}$ atom with a nuclear spin $I=\frac{3}{2}$. The observed quadrupole components for several transitions are listed in Table III. Splittings of the observed transitions were fit by a least-squares procedure to the parameters $e Q q_{a}=\chi_{a a}$ and $e Q q_{a} \eta=\chi_{b b}-\chi_{c c}$ using Ref. (7, Eqs. (6-1), (6-19)) and data calculated from the Beaudet asymmetric rotor program (8). First-order corrections were satisfactory for fitting the observed spectrum. The coupling constants are listed in Table IV. As a check on the analysis, the splitting for the $3_{03} \rightarrow 4_{04}$ transition was independently calculated using Ref. (7, Eq. (6-18)) and standard tables. That equation computes $q_{J}$ as a function of the $\chi^{\prime}$ 's, $E(\kappa)$, and $\partial E(\kappa) / \partial_{\kappa}$. 
TABLE II. Rotational Constants and Moments of Inertia of Arsabenzene.

$$
\begin{aligned}
A & =4871.03 \pm 0.18 \mathrm{MHz} \\
B & =2295.87 \pm 0.01 \mathrm{MHz} \\
\mathrm{C} & =1560.10 \pm 0.01 \mathrm{MHz} \\
\kappa & =-0.555549 \\
I_{a} & =103.751 \mu \AA^{2} \\
I_{b} & =220.124 \mu \AA^{2} \\
I_{c} & =323.939 \mu \AA^{2} \\
I_{c} & =I_{a}-I_{b}=0.064 \mu \AA^{2}
\end{aligned}
$$

Dipole moment. The molecular dipole moment was determined by observation and analysis of the second-order Stark effect. Stark measurements were made at low electric field values (shifts between 2 and $5 \mathrm{MHz}$ ) so that the "weak field" approximation could be applied. In this approximation, the Stark effect is treated as a small perturbation on the quadrupole energy. A particular level is then characterized by the set of good quantum numbers $J, I, F$, and $M_{F}$. Equation (10-30) in Ref. (7) was used to calculate the Stark shifts for an energy level. The value of $0.7152 \mathrm{D}$ for OCS was used to calibrate the electric field.

Table V lists the experimental measurements of the second-order Stark effect as well as the total dipole moment of arsabenzene which lies coincident with the $a$ principal axis $\left(\mu_{b}=\mu_{c}=0\right.$, by symmetry). The uncertainty in the dipole moment is the standard deviation when computed from the four Stark coefficients in Table V. The agreement with the calculated Stark coefficients is not as good as normally expected by this technique but it appears satisfactory in view of the small shifts observed and the difficulty of observing clear, resolved Stark components for transitions of weak intensity with an overlapping hyperfine component.

\section{ANALYSIS AND DISCUSSION}

Structure. Two observations can be made from the spectral analysis. First, the expected $C_{2 v}$ symmetry of the molecule is affirmed by the apparent lack of $\mu_{b}$ and $\mu_{c}$ dipole components. Second, the small positive value of the inertial defect, $I_{c}-I_{b}-I_{a}$

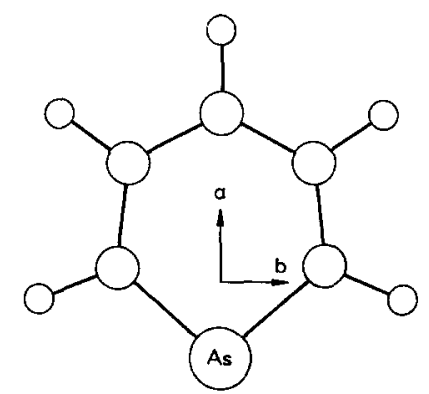

FIG. 1. Principal axes orientation in arsabenzene; $c$ axis perpendicular to $a$ and $b$ axes. 
TABLE III. Hyperfine Splitting in Arsabenzene (frequencies in MHz).

\begin{tabular}{|c|c|c|c|}
\hline Transition & $F+F^{\prime}$ & $\begin{array}{l}v-v^{a} \\
(o b s) \\
\end{array}$ & $\begin{array}{c}v^{-} y_{0} \\
\text { cale) }\end{array}$ \\
\hline${ }^{3} 03^{-4} 04$ & $\begin{array}{l}5 / 2+7 / 2 \\
7 / 2+9 / 2 \\
9 / 2+11 / 2\end{array}$ & $\begin{array}{r}5.25 \\
-\quad 0.17 \\
2.54\end{array}$ & $\begin{array}{r}5.20 \\
-0.25 \\
2.58\end{array}$ \\
\hline${ }^{3} 22^{-4} 23$ & $\begin{array}{l}5 / 2+7 / 2 \\
7 / 2+9 / 2 \\
9 / 2+11 / 2\end{array}$ & $\begin{array}{r}5.53 \\
-12.15 \\
7.00\end{array}$ & $\begin{array}{r}-5.46 \\
-12.16 \\
6.95\end{array}$ \\
\hline${ }^{3} 21^{-4} 22$ & $\begin{array}{l}3 / 2+5 / 2 \\
5 / 2+7 / 2 \\
7 / 2+9 / 2 \\
9 / 2 \rightarrow 11 / 2\end{array}$ & $\begin{array}{r}11.92 \\
-\quad 4.18 \\
-10.46 \\
5.77\end{array}$ & $\begin{array}{r}12.03 \\
-\quad 4.25 \\
-10.50 \\
5.78\end{array}$ \\
\hline${ }^{4} 23^{-5} 24$ & $\begin{array}{r}5 / 2 \rightarrow 7 / 2 \\
7 / 2 \rightarrow 9 / 2 \\
9 / 2 \rightarrow 11 / 2 \\
11 / 2 \rightarrow 13 / 2\end{array}$ & $\begin{array}{r}5.74 \\
-\quad 4.25 \\
-\quad 5.74 \\
4.27\end{array}$ & $\begin{array}{r}5.78 \\
-4.26 \\
-\quad 5.78 \\
4.26\end{array}$ \\
\hline $4_{32}-533$ & $\begin{array}{r}5 / 2 \rightarrow 7 / 2 \\
7 / 2 \rightarrow 9 / 2 \\
9 / 2 \rightarrow 11 / 2 \\
11 / 2 \rightarrow 13 / 2\end{array}$ & $\begin{array}{r}14.56 \\
-\quad 6.04 \\
-13.02 \\
7.53\end{array}$ & $\begin{array}{r}14.53 \\
-\quad 6.01 \\
-13.01 \\
7.53\end{array}$ \\
\hline${ }^{4} 31^{-5} 32$ & $\begin{array}{r}5 / 2 \rightarrow 7 / 2 \\
7 / 2 \rightarrow 9 / 2 \\
9 / 2 \rightarrow 11 / 2 \\
11 / 2 \rightarrow 13 / 2\end{array}$ & $\begin{array}{r}13.96 \\
-5.68 \\
-12.47 \\
7.17\end{array}$ & $\begin{array}{r}13.90 \\
-\quad 5.66 \\
-12.46 \\
7.19\end{array}$ \\
\hline${ }^{4} 13^{-5} 14$ & $\begin{array}{r}5 / 2 \rightarrow 7 / 2 \\
7 / 2 \rightarrow 9 / 2 \\
9 / 2 \rightarrow 11 / 2 \\
11 / 2 \rightarrow 13 / 2\end{array}$ & $\begin{array}{r}1.25 \\
-\quad 2.74 \\
-\quad 1.84 \\
2.17\end{array}$ & $\begin{array}{r}1.30 \\
-\quad 2.73 \\
-1.87 \\
2.16\end{array}$ \\
\hline${ }^{4} 22^{-5} 23$ & $\begin{array}{r}5 / 2 \rightarrow 7 / 2 \\
7 / 2 \rightarrow 9 / 2 \\
9 / 2 \rightarrow 11 / 2 \\
11 / 2 \rightarrow 13 / 2\end{array}$ & $\begin{array}{r}4.51 \\
-\quad 3.09 \\
-\quad 4.47 \\
3.20\end{array}$ & $\begin{array}{r}4.51 \\
-\quad 3.08 \\
-4.43 \\
3.16\end{array}$ \\
\hline${ }^{5} 24^{-6^{-b}}$ & $\begin{array}{r}7 / 2 \rightarrow 9 / 2 \\
13 / 2 \rightarrow 15 / 2 \\
9 / 2 \rightarrow 11 / 2 \\
11 / 2 \rightarrow 13 / 2\end{array}$ & $\begin{array}{r}2.97 \\
2.97 \\
-\quad 3.23 \\
-3.23\end{array}$ & $\begin{array}{r}3.07 \\
2.93 \\
-\quad 3.14 \\
-\quad 3.28\end{array}$ \\
\hline${ }^{5} 23^{-6} \frac{\mathrm{b}}{24}$ & $\begin{array}{rr}7 / 2 \rightarrow & 9 / 2 \\
13 / 2+15 / 2 \\
9 / 2 \rightarrow 11 / 2 \\
11 / 2 \rightarrow 13 / 2\end{array}$ & $\begin{array}{r}2.08 \\
2.08 \\
-2.25 \\
-2.25\end{array}$ & $\begin{array}{r}2.19 \\
2.04 \\
-\quad 2.17 \\
-\quad 2.32\end{array}$ \\
\hline
\end{tabular}

$a v_{0}$ frequencies listed in Table II.

b These transitions were not used to determine the quadrupole constants since some components were not resolved.

$=0.064 \mu \AA^{2}$, is typical for molecules of this type and is strongly supportive of a planar conformation. It is not possible to determine unambiguously a more detailed structure from the rotational constants since there are more structural parameters than experimental data. It is possible to estimate the $\mathrm{As}-\mathrm{C}$ bond length and the $\mathrm{C}-\mathrm{As}-\mathrm{C}$ angle by making suitable assumptions for the other structural parameters. However, there was little reason to undertake this in view of the excellent agreement with rotational constants calculated from the ED structure (4). Therefore the observed rotational constants are consistent with and support the structural conclusions in that report. 
TABLE IV. ${ }^{75}$ As Quadrupole Coupling Constants for Arsabenzene.

$$
\begin{aligned}
x_{\mathrm{aa}} & =e q_{\mathrm{a}}=-186.4 \pm 0.1 \mathrm{MHz} \\
x_{\mathrm{bb}} & =43.5 \pm 0.2 \mathrm{MHz} \\
\chi_{\mathrm{cc}} & =142.9 \pm 0.2 \mathrm{MHz} \\
\eta & =\frac{x_{\mathrm{bb}}-x_{\mathrm{cc}}}{x_{\mathrm{aa}}}=0.533 \pm 0.002
\end{aligned}
$$

Dipole moment. The dipole moment for arsabenzene of $1.10 \mathrm{D}$ can be compared with the values of $1.54 \mathrm{D}$ for phosphabenzene $(9)$ and $2.24 \mathrm{D}$ for pyridine (10). The direction of the dipole moments in these compounds is not directly available from the experimental data. A CNDO calculation $(6 a)$ concluded that the negative end of the dipole moment is pointed to the heteroatom in all three cases, although only in pyridine does the heteroatom have a residual negative charge. In phosphabenzene and arsabenzene residual charges on the $\alpha$ and $\beta$ carbon atoms weighed heavily in determining the direction of the dipole moment. The computed dipole moments in this calculation $(0.74 \mathrm{D}$ arsabenzene, $0.94 \mathrm{D}$ phosphabenzene, and $2.14 \mathrm{D}$ pyridine) are lower than the actual values but parallel the observed trend. The observed value of the dipole moment does not compare favorably with the value of $3.18 \mathrm{D}$ obtained in the ab initio MO calculation $(6 b)$. Inadequacies in the basis set were suggested as a cause of the discrepancy.

Nuclear quadrupole coupling constants. The coupling constants in Table IV due to the ${ }^{75}$ As nucleus provide information about the electric field gradients about the As atom along the principal inertial axes (Fig. 1). The interpretation of such data has been extensively discussed; the recent discussions by Gordy and Cook (11) and Lucken (12) are excellent summaries of many aspects.

A semiempirical formula for interpreting nuclear quadrupole coupling constants (NQCC) was first suggested by Townes and Daily (13). They assumed that the field gradient at a nucleus arises entirely from the electron population in the valence $p$

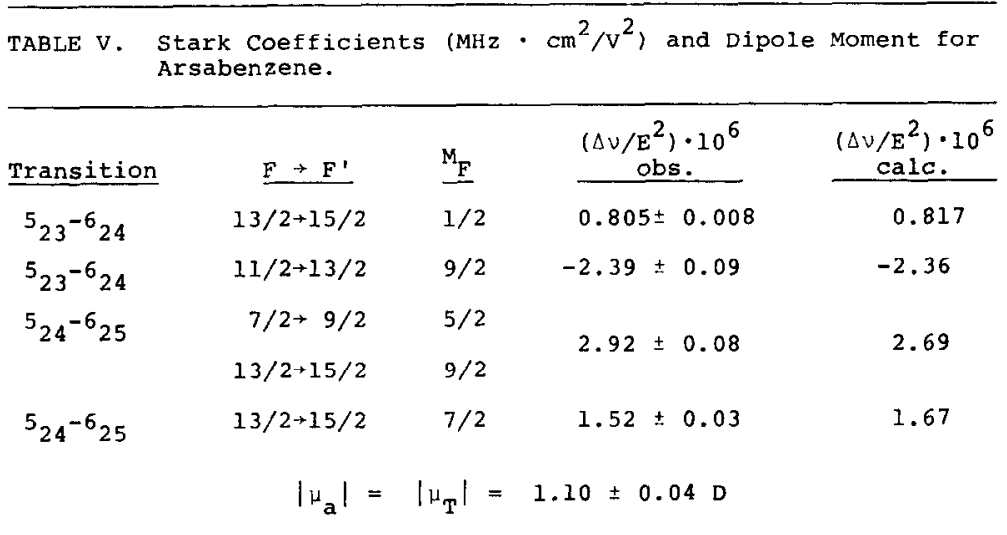


orbitals on that atom. Their formula, often called the Townes and Daily equation (12), is

$$
q_{z}=\left[n_{z}-\left(n_{x}+n_{y}\right) / 2\right] q^{0}
$$

where $q_{z}$ is the field gradient along the $z$ axis in the molecule, $q^{0}$ is the field gradient for one $p$ electron in the isolated atom and $n_{x}, n_{y}$, and $n_{z}$ are the electron populations of the respective $p$ orbitals in the molecule. This equation implies that $d$ orbitals and external charges (nuclear and electronic) do not contribute to the NQCC except indirectly as they affect the populations of the $p$ orbitals. This appears to be a good approximation for heavier elements $(11-15)$.

From Eq. (1), the data in Table IV, and the value of $q^{0}=-400 \mathrm{MHz}$ estimated for arsenic (11), several relationships are obtained:

$$
\begin{aligned}
& n_{a}-\left(n_{b}+n_{c}\right) / 2=0.47 \\
& n_{b}-\left(n_{a}+n_{c}\right) / 2=-0.11 \\
& n_{c}-\left(n_{a}+n_{b}\right) / 2=-0.36
\end{aligned}
$$

or

$$
n_{a}-n_{b}=0.387, \quad n_{a}-n_{c}=0.553, \quad n_{b}-n_{c}=0.166
$$

From symmetry, $n_{a}, n_{b}$, and $n_{c}$ refer to the $4 p$ orbitals oriented along the principal axes (Fig. 1). From these equations it is seen that $n_{a}>n_{b}>n_{c}$ but individual values cannot be obtained since only two equations are independent. Values might be calculated using a hybridized orbital scheme as was previously done for pyridine $(11,12)$. To prove tractable, this procedure must neglect $d$ orbital participation, a more questionable assumption in this case. ${ }^{3}$ We will limit discussion to some remarks regarding the relative values of $n_{a}, n_{b}$, and $n_{c}$.

The fact that $n_{a}$ has the largest value indicates that there is an excess of electron charge in the $p$ orbital along the $a$ axis relative to the other two axes. This would be consistent with that orbital participating in the lone pair orbital on the arsenic. Similarly, since $n_{c}$ has the smallest value, a deficiency of charge in the out-of-plane $p$ orbital relative to the in-plane $p$ orbitals is indicated. Furthermore, it seems safe to conclude that at least $n_{c}<1$ and that there is therefore a donation of charge to the other ring atoms through the $p_{\pi}=p_{c}$ orbital. If $n_{c}>1$ (which implies that $n_{a}$ and $n_{b}$ are also $>1$ ), a negative charge on arsenic would result. This is unlikely since arsenic is electropositive relative to carbon.

Although this analysis indicates that the out-of-plane $p$ orbital is a donor of electrons to the $\pi$ system, one must be cautious before assuming that arsenic is a $\pi$ donor overall; the effect of other orbitals must be considered, in particular the $4 d$ orbitals on the arsenic. Participation of these orbitals would return electron density to the arsenic.

${ }^{3}$ If $d$ orbitals are neglected and the procedure in Ref. (11) is followed, the following orbital populations can be obtained. $p_{a}=1.06 \pm 0.09, p_{b}-0.74 \pm 0.07, p_{c}=0.58 \pm 0.08$, and $n_{s}=1.66 \pm 0.01$ when $a_{s}{ }^{2}$ is assumed to be $0.127 \pm 0.015$. These values seem plausible in several respects but indicate a suspiciously large positive charge on arsenic $(0.9 \pm 0.2)$. One difficulty arises in choosing $a_{a^{2}}$ which is related to the amount of $s$ character in the hybrids. The two extreme values for $a_{2}{ }^{2}$ indicated above are obtained if either the CAsC angle is used to estimate it or an indirect procedure based on the electronegativity difference between $\mathrm{C}$ and $\mathrm{As}(11, \mathrm{Eq}$. (14.93)). However, the $\mathrm{CAsC}$ angle may not be a reliable gauge of $a_{8}{ }^{2}$ even neglecting $d$ orbitals in the hybrids (cf. $(11$, p. 607) or (12, p. 234)). 
To gain additional insights on the $\pi$ system, as well as the $\sigma$ system, the results of MO calculations can be examined. Neither the CNDO nor the ab initio calculations (6) reported individual orbital populations permitting direct comparison. However, both analyses concluded that arsenic donates electrons in the $\sigma$ system but accepts electrons in the $\pi$ system. This would be consistent with our premise that $n_{c}<1$ only if there is sizable participation by the $d$ orbitals. On the other hand, EHMO calculations (described in the Appendix) indicated both $\sigma$ and $\pi$ donation occurring with $n_{n}>n_{b}>n_{c}$. However, the EHMO calculations did not give good agreement with all the observed NQCC (typical values were $\chi_{a a}=-283 \mathrm{MHz}, \chi_{b b}=127 \mathrm{MHz}$, and $\chi_{c c}=156 \mathrm{MHz}$ ). Also, the order of some of the energy levels disagreed with the tentative assignment based on the photoelectron spectrum (5).

In summary, the observed NQCC indicate that $n_{a}>n_{b}>n_{c}$. Considering that arsenic is electropositive relative to carbon, at least $n_{c}<1$, which implies that the $p_{x}$ orbital donates electrons to the ring atoms. Semiempirical EHMO calculations qualitatively support these conclusions and indicate that arsenic is an overall $\pi$ donor; however, the preliminary CNDO report and the ab initio calculation indicate arsenic is an overall $\pi$ acceptor. Further investigations of the electronic structure of arsabenzene and tests of the validity of the Townes and Daily equation, Eq. (1), will be needed to resolve this question.

\section{APPENDIX: EHMO CALCULATIONS}

The structure reported in Ref. (4) was used. Matrix elements were generated by the formula $H_{i j}=0.5 K\left(H_{i i}+H_{j j}\right) S_{i j}$ with $K=1.75$. The orbital parameters chosen are listed in Table VI. The VOIP for the As $4 d$ is the parameter most difficult to choose and the EHMO numerical results are very dependent on its value. The value in Table VI was chosen using a procedure outlined by Wong (16). The orbital populations of interest $\left(N_{4 s, p, d}\right)$ were $s=1.54, p_{a}=1.34, p_{b}=0.65, p_{c}=0.60, d_{c} 2 \approx 0, d_{b c}=0.10, d_{a c}$ $=0.06, d_{a b} \approx 0, d_{b^{2}-a^{2}}=0.03$. If the VOIP for the arsenic $4 d$ was changed to $-7.5 \mathrm{eV}$, their participation becomes more extensive but still not quite sufficient to make arsenic a $\pi$ acceptor. The orbital populations of interest are $s=1.55, p_{a}=1.32, p_{b}=0.62$, $p_{c}=0.52, d_{c}^{2} \approx 0, d_{b c}=0.23, d_{a c}=0.16, d_{a b}=0.05$, and $d_{b^{2}-a^{2}}=0.14$.

TABLE VI. Orbital Parameters for the EHMO Calculations.

\begin{tabular}{cccc}
\hline Atom & $\begin{array}{c}\text { Atomic } \\
\text { Orbital }\end{array}$ & $\begin{array}{c}\text { Exponent } \\
\text { VoIP } \\
\text { H }\end{array}$ & $1 \mathrm{eV})$ \\
$\mathrm{C}$ & $2 \mathrm{~s}$ & 1.200 & -13.60 \\
& $2 \mathrm{p}$ & 1.608 & -19.42 \\
$\mathrm{As}$ & $4 \mathrm{~s}$ & 1.568 & -10.64 \\
& $4 \mathrm{p}$ & 2.236 & -17.95 \\
& $4 \mathrm{~d}$ & 1.862 & -9.19 \\
& & 1.300 & -5.9 \\
\hline
\end{tabular}




\section{ACKNOWLEDGMENTS}

This work was supported by grants GP-38750X and GP-30589X from the National Science Foundation. The assistance of Mr. Bruce Garner in preparing the compound and helpful discussions with Professor L. S. Bartell are gratefully acknowledged.

RECEIVED : October 30, 1974

\section{REFERENCES}

1. A. J. AsHe III, J. Am. Chem. Soc. 93, 3293 (1971).

2. A. J. Aste III, J. Am. Chem. Soc. 93, 6690 (1971).

3. A. J. Ashe III AND M. D. Gordon, J. Am. Chem. Soc. 94, 7596 (1972).

4. T. C. Wong, A. J. Ashe III, And L. S. Barteld, J. Mol. Struct. 25, 65 (1975).

5. C. Batich, E. Heilbronner, V. Hornung, A. J. Ashe III, D. T. Clark, U. T. Cobley, D. Kilcast, and I. Scanlan, $J$. Am. Chem. Soc. 95, 928 (1973).

6. (a) H. L. Hase, A. Schweig, H. HuHn, aNd J. Radlory, Telrahedron 29, 475 (1973).

(b) D. T. Clark and I. W. Scanlon, J. Chem. Soc., Farad. Trans. II. 70, 1222 (1974).

7. C. H. Townes and A. L. Schawlow, "Microwave Spectroscopy," Chap. 6, McGraw-Hill, New York, 1955.

8. R. BEAUDET, Ph.D. thesis, Harvard University, 1961.

9. R. L. Kuczxowski and A. J. AsHe III, J. Mol. Spectrose. 42, 457 (1972).

10. G. O. S $\varnothing$ Rensen, L. Mahler, AND N. Rastrup-ANDERson, J. Mol. Struct. 20, 119 (1974).

11. W. Gordy AND R. L. Cook, in "Chemical Applications of Spectroscopy," Vol. IX (W. West, Ed.), Chap. 14, Interscience, New York, 1970.

12. E. A. C. Lucken, "Nuclear Quadrupole Coupling Constants," Academic Press, London, 1969.

13. C. H. Townes and B. P. DaIlex, J. Chem. Phys. 17, 782 (1949).

14. F. A. Cotton and C. B. Harris, Proc. Nal. Acad. Sci. U. S. 56, 12 (1966).

15. W. D. White and R. S. Drago, J. Chem. Phys. 52, 4717 (1970).

16. T. C. Wong, Ph.D. thesis, University of Michigan, 1974. 